\title{
The Explicit Solution and Precise Distribution of CKLS Model under Girsanov Transform
}

\author{
Yunjiao $\mathrm{Hu}^{1}$, Guangqiang Lan $^{1}$ \& Chong Zhang ${ }^{1}$ \\ ${ }^{1}$ School of Science, Beijing University of Chemical Technology, Beijing 100029, China \\ Correspondence: Guangqiang Lan, School of Science, Beijing University of Chemical Technology, Beijing 100029, \\ China. E-mail: langq@ mail.buct.edu.cn
}

Received: November 27, 2014 Accepted: December 27, 2014 Online Published: January 8, 2015

doi:10.5539/ijsp.v4n1p68 URL: http://dx.doi.org/10.5539/ijsp.v4n1p68

Supported by China Scholarship Council, National Natural Science Foundation of China (NSFC11026142) and Beijing Higher Education Young Elite Teacher Project (BJYC34).

\begin{abstract}
The relation between CKLS model and CIR model will be investigated in this paper. It will be shown that under a suitable transformation, any CKLS model of order $\frac{1}{2}<\gamma<1$ or $\gamma>1$ corresponds to a CIR model under a new probability space. Moreover, the explicit solution and the precise distribution of the CKLS model at any time $t$ are obtained under the new probability space. The moment estimation of CKLS model will be given finally.
\end{abstract}

Keywords: interest rate; CKLS model; CIR model; Girsanov transform; martingale.

\section{Introduction}

Suppose that $\left(\Omega, \mathscr{F}, P ;\left(\mathscr{F}_{t}\right)_{t \geq 0}\right)$ is a right continuous filtered probability space satisfying the usual conditions. In 1992, Chan, Karolyi, Longstaff and Sanders (Chan, Karolyi, Longstaff \& Sanders, 1992) suggested modelling the behavior of the instantaneous interest rate by the following stochastic differential equation

$$
d r_{t}=\left(a-b r_{t}\right) d t+\sigma r_{t}^{\gamma} d B_{t}
$$

where the initial interest rate $r_{0}>0, a>0, b \in \mathbb{R}, \sigma>0$ and $\gamma \geq \frac{1}{2} . B_{t}$ denotes an 1-dimensional standard $\mathscr{F}_{t}$-Brownian motion.

This so called CKLS model is a very important model in both theory and application, it contains many important models in finance. These models can be obtained from (1) by simply placing the appropriate restrictions on the four parameters $a, b, \sigma$ and $\gamma$. For example, if $\gamma=0$, it becomes to a Vasicek model; $\gamma=1, a=0$ a geometric Brownian motion; $\gamma=\frac{1}{2}$ a CIR model, etc..

There are many literatures concerned about the solutions as well as other properties of CKLS model. For example, Choi and Wirjanto (Choi \& Wirjanto 2007) presented an analytic approximation formula for pricing zero-coupon bonds under CKLS models, Tangman, Thakoor, Dookhitram and Bhuruth (Tangman, Thakoor, Dookhitram \& Bhuruth, 2011) considered fast approximations of bond option prices under CKLS models, while Tang and Chen (Tang \& Chen, 2009) studied the parameter estimation and bias correction for diffusion processes which contain CKLS models as special cases, Khor, Pooi and $\mathrm{Ng}$ (Khor, Pooi \& Ng, 2013) studied the bond (European) option pricing under the CKLS model.

It is well known that there is a unique non negative solution of the equation (1). By Mao (Mao, 2007) Chapter 9, we also know that the solution $r_{t}>0$ for all $t \geq 0$ almost surely.

The motivation of this work is the following:

In Lamberton and Lapeyre (Lamberton \& Lapeyre, 1996), Proposition 6.2.5, the authors presented the Laplace transform of $r_{t}$ and $\int_{0}^{t} r_{s} d s$ in the case of CIR model (that is, $\gamma=\frac{1}{2}$ ), and finally they obtained the pricing formula of zero-coupon bond and the bond option. So a natural question raises: if we can transform CKLS model into a CIR model, then we can use the known results of CIR model to the CKLS model. 
In the following, we assume that $r_{t}$ is the unique positive solution of SDE (1).

Our main result is the following theorem.

Theorem 1 Let $\gamma>1$, or $\gamma \in\left(\frac{1}{2}, 1\right)$ and $\frac{\gamma}{\sigma} \geq 1, b>0$. Define $f(x)=\frac{C^{2}}{4(1-\gamma)^{2}} x^{2(1-\gamma)}$. Then there exist a new filtered probability space $\left(\Omega, \mathscr{F}, Q ;\left(\mathscr{F}_{t}\right)_{t \geq 0}\right)$ and a $Q$ Brownian motion $\bar{B}_{t}$ such that $Y_{t}=f\left(r_{t}\right)$ satisfies the following CIR model:

$$
d Y_{t}=\left(\frac{\sigma^{2} C^{2}}{4}+2 b(1-\gamma) Y_{t}\right) d t+\sigma C \sqrt{Y_{t}} d \bar{B}_{t}
$$

Remark 1 When $\gamma \in\left(\frac{1}{2}, 1\right)$ and $\frac{\gamma}{\sigma} \geq 1, b>0$, equation (2) is not the usual mean reverting square root process any more since the coefficient of $Y_{t}$ is $2 b(1-\gamma)>0$.

In general, we can not solve equation (1) explicitly except for some special cases (e.g., $\gamma=1)$, but by a suitable measure transform, we can get the explicit solution of our CKLS model under the new probability space.

Theorem 2 Assume that $\gamma>1$, or $\gamma \in\left(\frac{1}{2}, 1\right)$ and $\frac{\gamma}{\sigma} \geq 1, b>0$. Under the probability measure $Q$ (which is the same as in Theorem 1), the explicit solution of equation (1) is

$$
r_{t}=\left|r_{0}^{1-\gamma} e^{-(\gamma-1) b t}+\sigma\right| \gamma-1\left|\int_{0}^{t} e^{-b(\gamma-1)(t-s)} d \bar{B}_{s}\right|^{\frac{1}{1-\gamma}} .
$$

We can also get the distribution of $r_{t}$.

Theorem 3 Assume that $\gamma>1$, or $\gamma \in\left(\frac{1}{2}, 1\right)$ and $\frac{\gamma}{\sigma} \geq 1, b>0$. Under the probability measure $Q$, the precise density function of the solution $r_{t}$ of equation (1) is

$$
g_{t}(x)=g_{\delta, \zeta}(f(x) / L)\left|f^{\prime}(x)\right| / L, \forall x>0,
$$

where $f$ is the same as in Theorem $1, g_{\delta, \zeta}$ is the density of the non-central chi-square law with $\delta$ degrees of freedom and parameter $\zeta$,

$$
\delta=C^{2}, L=\frac{\sigma^{2} C^{2}}{8 b(\gamma-1)}\left(1-e^{2 b(1-\gamma) t}\right), \zeta=\frac{8 Y_{0} b(\gamma-1)}{\sigma^{2} C^{2}\left(e^{2 b(\gamma-1) t}-1\right)} .
$$

Finally, let us consider the moment estimation of $r_{t}$. We have

Theorem 4 Assume that $1<\gamma \leq \frac{3}{2}$, or $\frac{1}{2} \leq \gamma<1$ and $(2 \gamma+1) \sigma^{2} \leq 2 a$. Then

$$
\mathbb{E} \int_{0}^{t} r_{s}^{-2 \gamma} d s \vee \mathbb{E} \int_{0}^{t} r_{s}^{2(\gamma-1)} d s<\infty, \forall t>0,
$$

where $a \vee b:=\max (a, b)$.

The rest of the paper is organized as follows. Firstly, we study the relation between CKLS model and CIR model in Section 2, we derive the transform under which an arbitrary CKLS model can be transformed to a CIR model formally. In Section 3 we will prove that the condition of Girsanov transform is satisfied (that is, $R_{t}$ defined in Section 2 is a true martingale under $Q$ ). After this, we prove Theorem 2 and Theorem 3 in Section 4 . Finally we give the moment estimation of CKLS model, which is our Theorem 4.

\section{The Relation between CKLS Model and CIR Model}

Suppose $f: \mathbb{R}_{+} \rightarrow \mathbb{R}_{+}$is a differentiable function such that

$$
x^{\gamma} f^{\prime}(x)=C \sqrt{f(x)}, C>0 .
$$

Then we have

$$
f(x)=\frac{C^{2}}{4(1-\gamma)^{2}}\left(x^{1-\gamma}+C^{\prime}\right)^{2},
$$

where $C^{\prime}$ is an arbitrary contant. Take $C^{\prime}=0$ for simplicity. Then

$$
f(x)=\frac{C^{2}}{4(1-\gamma)^{2}} x^{2(1-\gamma)} .
$$


We also have

$$
f^{\prime}(x)=\frac{C^{2}}{2(1-\gamma)} x^{1-2 \gamma}, \quad f^{\prime \prime}(x)=\frac{C^{2}(1-2 \gamma)}{2(1-\gamma)} x^{-2 \gamma}
$$

It's clear that $f(x)$ is strictly monotone on $(0, \infty)$, so we have

$$
f^{-1}(x)=\left|\frac{2(\gamma-1)}{C}\right|^{\frac{1}{1-\gamma}} x^{\frac{1}{2(1-\gamma)}}
$$

By Itô's formula, we have

$$
\begin{aligned}
d f\left(r_{t}\right) & =\left[f^{\prime}\left(r_{t}\right)\left(a-b r_{t}\right)+\frac{\sigma^{2}}{2} f^{\prime \prime}\left(r_{t}\right) r_{t}^{2 \gamma}\right] d t+\sigma f^{\prime}\left(r_{t}\right) r_{t}^{\gamma} d B_{t} \\
& =\left[\frac{a C^{2}}{2(1-\gamma)} r_{t}^{1-2 \gamma}+\frac{b C^{2}}{2(1-\gamma)} r_{t}^{2-2 \gamma}+\frac{\sigma^{2}}{2} \frac{C^{2}(1-2 \gamma)}{2(1-\gamma)}\right] d t+\sigma C \sqrt{f\left(r_{t}\right)} d B_{t}
\end{aligned}
$$

Denote $Y_{t}=f\left(r_{t}\right)$. Then $r_{t}=f^{-1}\left(Y_{t}\right)=\left|\frac{2(\gamma-1)}{C}\right|^{\frac{1}{1-\gamma}} Y_{t}^{\frac{1}{2(1-\gamma)}}$. Therefore,

$$
d Y_{t}=\left[\frac{\sigma^{2} C^{2}(1-2 \gamma)}{4(1-\gamma)}+\frac{b C^{2}}{2(1-\gamma)}\left(\frac{2(\gamma-1)}{C} \sqrt{Y_{t}}\right)^{2}+\frac{a C^{2}}{2(1-\gamma)} r_{t}^{1-2 \gamma}\right] d t+\sigma C \sqrt{Y_{t}} d B_{t}
$$

Define

$$
\begin{gathered}
q\left(r_{t}\right):=\left(\frac{a}{\sigma} r_{t}^{-\gamma}-\frac{\gamma \sigma}{2} r_{t}^{\gamma-1}\right) \operatorname{sgn}(\gamma-1), \\
\bar{B}_{t}:=B_{t}-\int_{0}^{t} q\left(r_{s}\right) d s
\end{gathered}
$$

and

$$
R_{t}:=\exp \left\{\int_{0}^{t} q\left(r_{s}\right) d B_{s}-\frac{1}{2} \int_{0}^{t} q\left(r_{s}\right)^{2} d s\right\}
$$

It's clear that $R_{t}$ is an $\mathscr{F}_{t}$ local martingale with respect to probability measure $P$. If $\left\{R_{t}\right\}$ is a real martingale with respect to $P$, then by Girsanov transform, there exists a probability measure $Q$ on $\Omega$ such that $\left.\frac{d Q}{d P}\right|_{\mathscr{F}_{t}}=R_{t}$ and $\bar{B}_{t}$ is an $\mathscr{F}_{t}$ Brownian motion with respect to $Q$. Then $Y_{t}$ satisfies the following CIR model

$$
d Y_{t}=\left(\frac{\sigma^{2} C^{2}}{4}+2 b(1-\gamma) Y_{t}\right) d t+\sigma C \sqrt{Y_{t}} d \bar{B}_{t}
$$

So the key point is to prove that $\left\{R_{t}\right\}$ is a true martingale, which will be fulfiled in the next Section.

\section{3. $R_{t}$ is a True Martingale with Respect to Probability $P$}

To prove that $R_{t}$ is a true martingale, let us consider the following auxiliary equation

$$
d \tilde{r}_{t}=\left(a-b \tilde{r}_{t}+q\left(\tilde{r}_{t}\right) \sigma \tilde{r}_{t}^{\gamma}\right) d t+\sigma \tilde{r}_{t}^{\gamma} d B_{t}^{\prime}, \tilde{r}_{0}=r_{0}
$$

where $B_{t}^{\prime}$ is Brownian motion, $q$ are defined as in Section 2. Since we have known that, with probability one, the solution of equation (1) will never leave the state space $(0, \infty)$, by Mijatović and Urusov (Mijatović \& Urusov, 2012) Corollary 2.2, we only need to prove that the solution of equation (5) will also never leave the state space $(0, \infty)$.

\subsection{In Case $\gamma>1$}

If $\gamma>1$, then equation (5) could be transformed to

$$
d \tilde{r}_{t}=\left(2 a-b \tilde{r}_{t}-\frac{\gamma \sigma^{2}}{2} \tilde{r}_{t}^{2 \gamma-1}\right) d t+\sigma \tilde{r}_{t}^{\gamma} d B_{t}^{\prime} .
$$


For this equation, since $\mu(x)=2 a-b x-\frac{\gamma \sigma^{2}}{2} x^{2 \gamma-1}$ satisfies

$$
(x-y)(\mu(x)-\mu(y)) \leq 0 \leq K(x-y)^{2}, \forall x, y
$$

and $x^{\gamma}$ is local Lipschitz continuous, by Gyöngy and Krylov (Gyöngy \& Krylov, 1996) Corollary 2.7, there exists a unique solution solution of equation (5). We now prove that the solution $\tilde{r}_{t}>0, \forall t \geq 0$ almost surely. Define

$$
\tau_{k}:=\inf \left\{t>0, \tilde{r}_{t} \notin\left(\frac{1}{k}, k\right)\right\}, k \geq k_{0}
$$

where $k_{0}$ is large enough such that $r_{0} \in\left(\frac{1}{k_{0}}, k_{0}\right)$.

We only need to prove

$$
\tau_{\infty}:=\lim _{k \rightarrow \infty} \tau_{k}=\infty .
$$

If not, we can choose $T>0,0<\varepsilon<1$ such that $P\left(\tau_{\infty} \leq T\right) \geq \varepsilon$. Then there exists $k_{1} \geq k_{0}$ such that

$$
P\left(\tau_{k} \leq T\right) \geq \varepsilon \quad \forall k \geq k_{1} .
$$

Define

$$
V(x)=\sqrt{x}-1-\frac{1}{2} \log x, x>0 .
$$

Then if $\tilde{r}_{t}>0$, Itô's formula yields that

$$
\begin{aligned}
d V\left(\tilde{r}_{t}\right)= & \frac{1}{2}\left(\tilde{r}_{t}^{-\frac{1}{2}}-\tilde{r}_{t}^{-1}\right)\left(2 a-b \tilde{r}_{t}-\frac{\gamma \sigma^{2}}{2} \tilde{r}_{t}^{2 \gamma-1}\right) d t \\
& +\frac{\sigma^{2}}{4}\left(-\frac{1}{2} \tilde{r}_{t}^{-\frac{3}{2}}+\tilde{r}_{t}^{-2}\right) \tilde{r}_{t}^{2 \gamma} d t+\frac{\sigma}{2}\left(\tilde{r}_{t}^{-\frac{1}{2}}-\tilde{r}_{t}^{-1}\right) \tilde{r}_{t}^{\gamma} d B_{t}^{\prime} \\
= & F\left(\tilde{r}_{t}\right) d t+\frac{\sigma}{2}\left(\tilde{r}_{t}^{-\frac{1}{2}}-\tilde{r}_{t}^{-1}\right) \tilde{r}_{t}^{\gamma} d B_{t}^{\prime},
\end{aligned}
$$

where

$$
F(x)=\frac{1}{2}\left(x^{-\frac{1}{2}}-x^{-1}\right)\left(2 a-b x-\frac{\gamma \sigma^{2}}{2} x^{2 \gamma-1}\right)+\frac{\sigma^{2}}{4}\left(-\frac{1}{2} x^{-\frac{3}{2}}+x^{-2}\right) x^{2 \gamma}
$$

Since the coefficients of the highest and lowest order of $F(x)$ are both negative (the coefficients are $-\frac{\sigma^{2}(2 \gamma+1)}{8},-a$, respectively), then $F(x)$ is bounded, say by $K$, on $x \in(0, \infty)$. Thus,

$$
d V\left(\tilde{r}_{t}\right) \leq K d t+\frac{\sigma}{2}\left(\tilde{r}_{t}^{-\frac{1}{2}}-\tilde{r}_{t}^{-1}\right) \tilde{r}_{t}^{\gamma} d B_{t}^{\prime}
$$

as long as $\tilde{r}_{t} \in(0, \infty)$. So we have

$$
\mathbb{E}\left(V\left(\tilde{r}_{T \wedge \tau_{k}}\right)\right) \leq V\left(r_{0}\right)+K T .
$$

Set $\Omega_{k}=\left\{\tau_{k} \leq T\right\}$, then $P\left(\Omega_{k}\right) \geq \varepsilon$ for $k \geq k_{1}$. Since

$$
\tilde{r}\left(\tau_{k}, \omega\right)=k \text { or } \frac{1}{k}
$$

then

$$
V\left(\tilde{r}\left(\tau_{k}, \omega\right)\right) \geq\left(\sqrt{k}-1-\frac{1}{2} \log k\right) \wedge\left(\frac{1}{2} \log k+\sqrt{\frac{1}{k}}-1\right) .
$$

Therefore,

$$
\begin{aligned}
V\left(r_{0}\right)+K T & \geq \mathbb{E}\left(V\left(\tilde{r}_{T \wedge \tau_{k}}\right)\right) \geq \mathbb{E}\left(1_{\Omega_{k}} V\left(\tilde{r}\left(\tau_{k}, \omega\right)\right)\right) \\
& \geq \varepsilon\left[\left(\sqrt{k}-1-\frac{1}{2} \log k\right) \wedge\left(\frac{1}{2} \log k+\sqrt{\frac{1}{k}}-1\right)\right] .
\end{aligned}
$$

Letting $k \rightarrow \infty$ leads to a contradiction

$$
\infty>V\left(r_{0}\right)+K T \geq \infty .
$$


So $\tau_{\infty}=\infty$. That is, with probability $1, r_{t}>0$ for all $t \geq 0$. Therefore, $R_{t}$ is a true martingale with respect to $P$. 3.2 In Case $\frac{1}{2} \leq \gamma<1$

In this case, equation (5) becomes to

$$
d \tilde{r}_{t}=\left(\frac{\gamma \sigma}{2} \tilde{r}_{t}^{2 \gamma-1}-b \tilde{r}_{t}\right) d t+\sigma \tilde{r}_{t}^{\gamma} d B^{\prime}{ }_{t}
$$

Note that the method we used in Subsection 3.1 to prove the positivity of the solution can not be used in the present case. Since

$$
\sigma x^{\gamma}>0, \forall x>0 \text { and } \frac{1}{\sigma^{2} x^{2 \gamma}}, \frac{\frac{\gamma \sigma}{2} x^{2 \gamma-1}-b x}{\sigma^{2} x^{2 \gamma}} \in L_{l o c}^{1}(0, \infty),
$$

where $L_{l o c}^{1}(0, \infty)$ denotes the class of locally integrable functions, i.e. the functions $(0, \infty) \rightarrow \mathbb{R}$ that are integrable on compact subsets of $(0, \infty)$. By (Engelbert \& Schmidt, 1985), (Engelbert \& Schmidt, 1991) or (Karatzas \& Shreve, 1991) Chapter 5, Theorem 5.15, there exists a unique in law weak solution that possibly exits its state space $(0, \infty)$.

We now prove that the solution $\tilde{r}_{t}>0, \forall t \geq 0$ almost surely by using Feller's test for explosions.

Define the scale function

$$
p(x)=\int_{1}^{x} \exp \left\{-2 \int_{1}^{y} \frac{\frac{\gamma \sigma}{2} z^{2 \gamma-1}-b z}{\sigma^{2} z^{2 \gamma}} d z\right\} d y .
$$

Compute

$$
p(x)=\exp \left\{-\frac{b}{\sigma^{2}(1-\gamma)}\right\} \int_{1}^{x} y^{-\frac{\gamma}{\sigma}} \exp \left\{\frac{b}{\sigma^{2}(1-\gamma)} y^{2(1-\gamma)}\right\} d y .
$$

In the case when $\frac{\gamma}{\sigma} \geq 1$ and $b>0$, it is ease to see that

$$
\lim _{x \downarrow 0} p(x)=-\infty \quad \text { and } \quad \lim _{x \uparrow \infty} p(x)=\infty .
$$

By (Karatzas \& Shreve, 1991) Proposition 5.22,

$$
P\left(\tau_{\infty}=\infty\right)=1,
$$

where $\tau_{\infty}$ is defined as Subsection 3.1. That is, the solution of equation will never leave the state space $(0, \infty)$. Therefore, $R_{t}$ is a true martingale. The proof of Theorem 1 is then complete.

\section{Explicit Solution and Precise Distribution of $r_{t}$ under Probability $Q$}

Proof of Theorem 2 By Theorem 1, we know that $Y_{t}=f\left(r_{t}\right)$ satisfies the CIR model. By Itô's formula

$$
\begin{aligned}
d \sqrt{Y_{t}} & =\frac{1}{2 \sqrt{Y_{t}}}\left[\left(\frac{\sigma^{2} C^{2}}{4}+2 b(1-\gamma) Y_{t}\right) d t+\sigma C \sqrt{Y_{t}} d \bar{B}_{t}\right]-\frac{1}{8 Y_{t}^{\frac{3}{2}}} \sigma^{2} C^{2} Y_{t} d t \\
& =b(1-\gamma) \sqrt{Y_{t}} d t+\frac{\sigma C}{2} d \bar{B}_{t} .
\end{aligned}
$$

So $\sqrt{Y_{t}}$ is an Ornstein-Uhlenbeck process, whose solution is

$$
\sqrt{Y_{t}}=\sqrt{Y_{0}} e^{b(1-\gamma) t}+\frac{\sigma C}{2} \int_{0}^{t} e^{b(1-\gamma)(t-s)} d \bar{B}_{s} .
$$

Here $\sqrt{x}$ should be understood as a real number $a$ such that $a^{2}=x$, otherwise we will get a contradiction since the left hand side of the equation is non negative while the right hand side will be negative with positive probability.

Thus

$$
r_{t}=\left|\frac{2(\gamma-1)}{C}\right|^{\frac{1}{1-\gamma}} Y_{t}^{\frac{1}{2(1-\gamma)}}=\left|r_{0}^{1-\gamma} e^{-(\gamma-1) b t}+\sigma\right| \gamma-1\left|\int_{0}^{t} e^{-b(\gamma-1)(t-s)} d \bar{B}_{s}\right|^{\frac{1}{1-\gamma}} .
$$

We complete the proof. 
Proof of Theorem 3 Define $L=\frac{\sigma^{2} C^{2}}{8 b(\gamma-1)}\left(1-e^{2 b(1-\gamma) t}\right)$. By (Lamberton \& Lapeyre, 1996) Proposition 6.2.5 with $\mu=0$, we know that $Y_{t} / L$ satisfies the non-central chi-square law with $\delta$ degrees of freedom and parameter $\zeta$, where

$$
\delta=C^{2}, \zeta=\frac{8 Y_{0} b(\gamma-1)}{\sigma^{2} C^{2}\left(e^{2 b(\gamma-1) t}-1\right)} .
$$

Thus the density of $Y_{t} / L$ is given by

$$
g_{\delta, \zeta}(x)=\frac{e^{-\zeta / 2}}{2 \zeta^{\delta / 4-1 / 2}} e^{-x / 2} x^{\delta / 4-1 / 2}\left(\frac{\sqrt{x \zeta}}{2}\right)^{\delta / 2-1} \sum_{n=0}^{\infty} \frac{(\sqrt{x \zeta} / 2)^{2 n}}{n ! \Gamma(\delta / 2+n)}, \quad x>0,
$$

where $\Gamma(x)=\int_{0}^{\infty} t^{x-1} e^{-t} d t$.

Therefore

$$
g_{t}(x)=g_{\delta, \zeta}\left(\frac{f(x)}{L}\right)\left|\frac{f^{\prime}(x)}{L}\right|, \quad x>0 .
$$

We then complete the proof of Theorem 3 .

Remark 2 Let us denote $P(t, T)$ the price of zero-coupon bond paying 1 dollar at a maturity date $T$. If we take the risk premium as $q\left(r_{t}\right)$, defined by (4), then $Q$ is the so-called risk neutral probability. Thus,

$$
\begin{aligned}
P(t, u) & =\mathbb{E}^{Q}\left(e^{-\int_{t}^{u} r_{s} d s} \mid \mathscr{F} t\right)=\mathbb{E}^{Q}\left(e^{-\int_{0}^{u-t} r_{s} d s}\right) \\
& =\mathbb{E}^{P}\left(e^{-\int_{0}^{u-t}\left|r_{0}^{1-\gamma} e^{-(\gamma-1) b s}+\sigma\right| \gamma-1\left|\int_{0}^{s} e^{-b(\gamma-1)(s-l)} d B_{l}\right|^{\frac{1}{1-\gamma}} d s}\right) .
\end{aligned}
$$

\section{Moment Estimations of $r_{t}$}

Proof of Theorem 4 By Itô's formula, it follows that

$$
\begin{aligned}
d r_{t}^{-2 \gamma} & =\left(-2 \gamma r_{t}^{-2 \gamma-1}\left(a-b r_{t}\right)+\frac{2 \gamma(2 \gamma+1) \sigma^{2}}{2} r_{t}^{-2}\right) d t-2 \gamma \sigma r_{t}^{-\gamma-1} d B_{t} \\
& =-2 a \gamma\left(r_{t}^{-2 \gamma}\right)^{1+\frac{1}{2 \gamma}} d t+2 b \gamma r_{t}^{-2 \gamma} d t+\gamma(2 \gamma+1) \sigma^{2}\left(r_{t}^{-2 \gamma}\right)^{\frac{1}{\gamma}} d t+M_{t} .
\end{aligned}
$$

5.1 Case I: $\frac{1}{2} \leq \gamma<1$

If $\frac{1}{2} \leq \gamma<1$, since

$$
\left(x^{-2 \gamma}\right)^{\frac{1}{\gamma}} \leq\left(x^{-2 \gamma}\right)^{1+\frac{1}{2 \gamma}}+1, \forall x>0
$$

we have

$$
\mathbb{E}\left(r_{t}^{-2 \gamma}\right) \leq r_{0}^{-2 \gamma}+\gamma(2 \gamma+1) \sigma^{2} t+2 b \gamma \int_{0}^{t} \mathbb{E}\left(r_{s}^{-2 \gamma}\right) d s
$$

in case $(2 \gamma+1) \sigma^{2} \leq 2 a$.

Gronwall's lemma yields that

$$
\mathbb{E}\left(r_{t}^{-2 \gamma}\right) \leq \Psi(t)+2 b \gamma \int_{0}^{t} \Psi(s) e^{2 b \gamma(t-s)} d s,
$$

where $\Psi(t)=r_{0}^{-2 \gamma}+\gamma(2 \gamma+1) \sigma^{2} t$. It's clear that the right hand side of the inequality is locally integrable with respect to $t$. Therefore,

$$
\mathbb{E} \int_{0}^{t} r_{s}^{-2 \gamma} d s<\infty
$$

holds for any $t>0$.

On the other hand, by Itô's formula,

$$
d r_{t}^{2(\gamma-1)}=\left(2(\gamma-1) r_{t}^{2 \gamma-3}\left(a-b r_{t}\right)+\frac{2(\gamma-1)(2 \gamma-3) \sigma^{2}}{2} r_{t}^{4 \gamma-4}\right) d t+2(\gamma-1) \sigma r_{t}^{3 \gamma-3} d B_{t}
$$


Notice that $2 \gamma-3 \leq 4 \gamma-4<0$, and if $(2 \gamma+1) \sigma^{2} \leq 2 a$, then

$$
\frac{2(\gamma-1)(2 \gamma-3) \sigma^{2}}{2} \leq 2 a(1-\gamma)
$$

Thus $\mathbb{E}\left(r_{t}^{2(\gamma-1)}\right) \leq r_{0}^{2(\gamma-1)}+(\gamma-1)(2 \gamma-3) \sigma^{2} t+2 b(1-\gamma) \int_{0}^{t} \mathbb{E}\left(r_{s}^{2(\gamma-1)}\right) d s$.

By using Gronwall's lemma again,

$$
\mathbb{E}\left(r_{t}^{2(\gamma-1)}\right) \leq \tilde{\Psi}(t)+2 b(1-\gamma) \int_{0}^{t} \tilde{\Psi}(s) e^{2 b(1-\gamma)(t-s)} d s,
$$

where $\tilde{\Psi}(t)=r_{0}^{2(\gamma-1)}+(\gamma-1)(2 \gamma-3) \sigma^{2} t$.

So we have proved Theorem 4 in case that $\frac{1}{2} \leq \gamma<1,(2 \gamma+1) \sigma^{2} \leq 2 a$.

5.2 Case II: $1<\gamma<\frac{3}{2}$

Note that $0<\frac{1}{\gamma}<1$ in this case. Since

$$
x^{\frac{1}{\gamma}} \leq x+1, \forall x>0
$$

by (10) we have

$$
\mathbb{E}\left(r_{t}^{-2 \gamma}\right) \leq r_{0}^{-2 \gamma}+\gamma(2 \gamma+1) \sigma^{2} t+\gamma\left(2 b+(2 \gamma+1) \sigma^{2}\right) \int_{0}^{t} \mathbb{E}\left(r_{s}^{-\gamma}\right) d s
$$

Gronwall's lemma yields that

$$
\mathbb{E}\left(r_{t}^{-2 \gamma}\right) \leq \Psi(t)+\gamma\left(2 b+(2 \gamma+1) \sigma^{2}\right) \int_{0}^{t} \Psi(s) e^{\gamma\left(2 b+(2 \gamma+1) \sigma^{2}\right)(t-s)} d s
$$

where $\Psi(t)=r_{0}^{-2 \gamma}+\gamma(2 \gamma+1) \sigma^{2} t$. Then

$$
\mathbb{E} \int_{0}^{t} r_{s}^{-2 \gamma} d s<\infty
$$

holds for any $t>0$.

On the other hand, by (1),

$$
\mathbb{E}\left(r_{t}\right)=r_{0}+a t-b \int_{0}^{t} \mathbb{E}\left(r_{s}\right) d s .
$$

Thus $\mathbb{E}\left(r_{t}\right)=\frac{a}{b}+\left(r_{0}-\frac{a}{b}\right) e^{-b t}$. Since $1<\gamma \leq \frac{3}{2}$,

$$
\mathbb{E}\left(r_{t}^{2(\gamma-1)}\right) \leq 1+\mathbb{E}\left(r_{t}\right)=1+\frac{a}{b}+\left(r_{0}-\frac{a}{b}\right) e^{-b t} .
$$

We complete the proof of Theorem 4.

From this, we know that $\mathbb{E} \int_{0}^{t} q\left(r_{s}\right)^{2} d s<\infty$ for any $t>0$ if $\frac{1}{2} \leq \gamma<1$ and $(2 \gamma+1) \sigma^{2} \leq 2 a$, or $1<\gamma<\frac{3}{2}$. Therefore, we have proved that $\int_{0}^{t} q\left(r_{s}\right) d B_{s}$ is a true martingale.

\section{Acknowledgement}

The second author would like to thank Professor Feng-Yu Wang, anonymous referee and editors for helpful comments and suggestions, which greatly improved the quality of this paper.

\section{References}

Chan, K., Karolyi, A., Longstaff, F., \& Sanders, A. (1992). An empirical comparison of alternative models of the short-term interest rate. The Journal of Finance, 47(3), 1209-1227. http://dx.doi.org/10.1111/j.15406261.1992.tb04011.x

Choi, Y., \& Wirjanto, T. (2007). An analytic approximation formula for pricing zero-coupon bonds. Finance Research Letters, 4, 116-126. http://dx.doi.org/10.1016/j.frl.2007.02.001 
Engelbert, H.-J., \& Schmidt, W. (1985). On one-dimensional stochastic differential equations with generalized drift. In M. Metivier and E. Pardoux, editors, Stochastic differential systems (Marseille-Luminy, 1984), volume 69 of Lecture Notes in Control and Inform. Sci., pp. (143-155). Springer, Berlin.

Engelbert, H.-J., \& Schmidt, W. (1991). Strong Markov continuous local martingales and solutions of onedimensional stochastic differential equations. III. Math. Nachr., 151,149-197. http://dx.doi.org/10.1002/mana.19911510111

Gyöngy, I., \& Krylov, N. (1996). Existence of strong solutions for Itô's stochastic equations via approximations. Probab. theory and relat. fields, 105, 143-158. http://dx.doi.org/10.1007/bf01203833

Karatzas, I., \& Shreve, S. (1991). Brownian motion and stochastic calculus, Second Edition. Springer-Verlag, Berlin, Heidelberg, Newyork.

Khor, C.Y., Pooi, A.H., \& Ng, K.H. (2013). Bond option pricing under the CKLS model. World Applied Sciences Journal, 21 (Special Issue of Applied Math), 150-155. http://dx.doi.org 10.5829/idosi.wasj.2013.21.am.21138

Lamberton, D., \& Lapeyre, B. (1996). Introduction to stochastic calculus applied to finance. Translated by Rabeau, N. and Mantion, F., Chapman \& Hall/CRC.

Mao, X. (2007). Stochastic differential equations and applications. Second edition, Horwood, Chichester.

Mijatović, A., \& Urusov, M. (2012). On the martingale property of certain local martingales. Probab. theory and relat. fields, 152, 1-20. http://dx.doi.org/10.1007/s00440-010-0314-7

Tang, C., \& Chen, S. (2009). Parameter estimation and bias correction for diffusion processes. Journal of Econometrics, 149, 65-81. http://dx.doi.org/10.1016/j.jeconom.2008.11.001

Tangman, D.Y., Thakoor, N., Dookhitram, K., \& Bhuruth, M. (2011). Fast approximations of bond option prices under CKLS models. Finance Research Letters, 8, 206-212. http://dx.doi.org 10.1016/j.frl.2011.03.002

\section{Copyrights}

Copyright for this article is retained by the author(s), with first publication rights granted to the journal.

This is an open-access article distributed under the terms and conditions of the Creative Commons Attribution license (http://creativecommons.org/licenses/by/3.0/). 